Boise State University

ScholarWorks

8-7-2013

\title{
Direct Observation of Self-Assembled Chain-Like Water Structures in a Nanoscopic Water Meniscus
}

Byung I. Kim

Boise State University

Ryan D. Boehm

Boise State University

Jeremy R. Bonander

Boise State University 


\title{
Direct Observation of Self-assembled Chain-like Water Structures in a Nanoscopic Water Meniscus
}

\author{
Byung I. Kim ${ }^{1}$, Ryan D. Boehm, and Jeremy R. Bonander
}

Author Affiliations

Boise State University, Department of Physics, Boise, Idaho, 83725, USA

${ }^{1}$ To whom correspondence should be addressed. E-mail: ByungKim@boisestate.edu

Keywords: chain-like water structure, freely jointed chain (FJC), self-assembly, viscosity 


\begin{abstract}
Sawtooth-like oscillatory forces generated by water molecules confined between two oxidized silicon surfaces were observed using a cantilever-based optical interfacial force microscope when the two surfaces approached each other in ambient environments. The humidity-dependent oscillatory amplitude and periodicity were 3-12 $\mathrm{nN}$ and 3-4 water diameters, respectively. Half of each period was matched with a freely jointed chain model, possibly suggesting that the confined water behaved like a bundle of water chains. The analysis also indicated that water molecules self-assembled to form chain-like structures in a nanoscopic meniscus between two hydrophilic surfaces in air. From the friction force data measured simultaneously, the viscosity of the chain-like water was estimated to be between $10^{8}$ and $10^{10}$ times greater than that of bulk water. The suggested chain-like structure resolves many unexplained properties of confined water at the nanometer scale, thus dramatically improving the understanding of a variety of water systems in nature.
\end{abstract}




\section{Introduction}

The apprehension of water's structure in local and confined spaces is extremely important for understanding the interfacial phenomena of water at nanometer scales. These phenomena include assembly and function of biomolecules, ${ }^{1-3}$ wetting and interfacial interactions, ${ }^{4-6}$ corrosion processes, ${ }^{7,8}$ condensation and crystallization of water vapor on surfaces, ${ }^{3,8-13}$ and amorphous solid water in an interstellar medium. ${ }^{14}$ It has long been postulated that water in confined spaces can have dramatically different physical properties from bulk water in extended spaces. ${ }^{15-22}$ Three-dimensional hydrogen networks in bulk water are disrupted at solid-liquid interfaces due to the confined geometries and the interaction of water with other materials. Water molecules next to other materials will rearrange to form confined water. In a confined space, such as a biological cell, this confined water is thought to behave differently from bulk water to a substantial degree. ${ }^{8,13,16,20-22}$ The layers of water in confined spaces affect the biological processes and functions of biomolecules (lipids, DNA, proteins, etc.) ${ }^{23-28}$ through the change of mechanical properties. ${ }^{3}$ These hydration layers also influence the chemical and mechanical properties of hydrophilic or hydrophobic surfaces such as oxides (silica, alumina, mica, clay, nano tubes, and graphite). ${ }^{6,19-22,29-34}$ Water is also a pervasive environmental component whose interactions with surfaces are critical to system performance in a wide range of emerging nano and other chemical and biological technologies.

The long-range electrostatic double-layer force, which depends strongly on ionic strength in water, has been well established with theoretical models, such as the DLVO (Derjaguin, Landau, Verwey, and

Overbeek) theory. ${ }^{35,36}$ However, the structure of confined water in air still remains widely unknown. This lack of understanding has led to a variety of observed phenomena which have not been explained. For one, environment scanning electron microscopy (SEM) studies show the meniscus heights in a 
nanoscale junction to frequently be far larger than what the Kelvin equation predicts. ${ }^{37-39}$ Although the effective Kelvin radii in their SEM images are strongly positive, water bridges remained highly stable in an ambient environment. ${ }^{38}$ Nanomechanical measurements have demonstrated that the viscosity of water confined between the tip and the surface in air are shown to be $\sim 10^{6}$ times larger than the viscosity of water in extended spaces or bulk water. ${ }^{40-42}$ Nanoscale studies found that the evaporation rate of capillary water was significantly less than that of bulk water, especially at low relative humidity (RH) values. $^{37,43}$ Another recent study has shown that nucleation timescales in the capillary meniscus formation were comparable to those for the ice evaporation at room temperature. ${ }^{44}$ Earlier kinetic measurements also found that the sublimation energy was associated with two hydrogen bonds breaking rather than 3.5-4 bonds. ${ }^{45}$ Water molecules were also reported to form elongated structures on surfaces in air. ${ }^{46-48}$ The viscous and sticky layers of water in ambient conditions were reported by Salmeron et al., ${ }^{4,9,10}$ by Jinesh and Frenken, ${ }^{40}$ and by Major et al. ${ }^{41,42}$ The unexplained phenomena underscore the fact that very little is known about the structure of confined water and its formation mechanism at the nanometer scale.

The ordering of water molecules confined at the nanometer scale next to hydrophilic surfaces has been extensively investigated by scanning probe techniques, such as atomic-force microscopy (AFM). ${ }^{10,44,49-52}$ However, most of these AFM studies were only able to provide indirect evidence of the existence of layered water confined at the nanometer scale. The AFM is unable to generate force variation as a function of the controlled distance between the tip and the surface, which makes its data difficult to analyze directly with existing theories due to the missing data points. An attempt to obtain an interfacial force measurement using the AFM is complicated by what is commonly referred to as the "snap-tocontact" problem: the uncontrolled movement of the probe to the surface when the intermolecular force gradient exceeds the spring constant of the AFM cantilever probe. A stiffer cantilever could avoid this 
uncontrolled movement, but the sensitivity of AFM measurement would be remarkably degraded. This has been a significant barrier to revealing the water structure in the nanoscopic water junction between the tip and the surface in ambient conditions through direct measurement of force-distance curves using the AFM. This limited ability highlights the need for a more accurate and direct approach.

To understand these mysterious properties of water in confined spaces, a newly developed cantileverbased optical interfacial force microscope (COIFM) ${ }^{47,53-56}$ was employed to probe confined water at the nanoscale. The COIFM uses a force-feedback technique to avoid the tip instabilities associated with AFM measurements, while still maintaining a high level of sensitivity. We observed remarkable oscillatory forces with amplitude of $60-90 \mathrm{nN}$ and periodicity of 3-4 water molecule diameters in an ambient environment between two silicon surfaces as the tip-sample distance decreased. ${ }^{47,54}$ The oscillatory forces decreased from large to progressively smaller as the humidity increased. ${ }^{47}$ The frequency of oscillatory forces increased with the existence of lateral tip-modulation and with the slower approaching tip-speed, suggesting that the oscillation resulted from kinetically activated processes. Here, to understand the fundamental origin of the oscillatory forces at the molecular level, we analyzed the upward force responses of large sawtooth-like oscillatory forces using a freely jointed chain (FJC) model. The result suggests that water formed self-assembled chain-like structures between two hydrophilic surfaces and multiple-layering transitions between structures in ambient environments.

\section{Experimental}

We measured normal force $\left(f_{\text {normal }}\right)$ and friction force $\left(f_{\text {friction }}\right)$ simultaneously in ambient environments as the probe approached an oxidized $\mathrm{Si}(100)$ substrate (SPI supplies, West Chester, PA), using the recently published COIFM method. ${ }^{47,53-59}$ The sample preparation, the experimental setup, and the determination of force conversion factor were detailed in our previous report. ${ }^{54}$ It is worth noting that the 
determination of the conversion factor requires the spring constant of the cantilever. This is because the force-voltage relationship was obtained while feedback was off. The force was found from the Hookes law by multiplying the cantilever bending (acted by an applied voltage to the $\mathrm{ZnO}\left(\mathrm{V}_{\mathrm{ZnO}}\right)$ ) with the nominal spring constant $(\sim 3 \mathrm{~N} / \mathrm{m})^{60}$. Since the force while feedback is off is equal to the counter force by the same voltage while feedback is on, the slope of the force-distance curve corresponds to the force conversion factor during the COIFM force-distance measurement. ${ }^{54}$

We also performed x-ray photoelectron spectroscopy (XPS) to ensure the absence of possible foreign impurities on the cleaned wafer surface (see more details in supplemental materials ${ }^{61}$ ). The tip radius of $\sim 10 \mathrm{~nm}$ provided by the manufacturer ${ }^{60}$ was verified by SEM. ${ }^{55}$ Before the measurements, the out-ofplane misalignment between the tip and the surface was corrected by leveling the sample surface with respect to the cantilever using the pitch-and-roll alignment setup of the COIFM head. The degree of the misalignment was evaluated by obtaining a topographic image. The pitch-and-roll alignment process and topographic imaging were repeated until satisfactory alignment of the sample plane was obtained. In this way, the tip can be aligned to the surface with $\pm 5^{\circ}$ accuracy, which is sufficient for the interpretation of the observed data. For the collection of force-distance curves, the deflection voltages $\left(\mathrm{V}_{\mathrm{A}-\mathrm{B}}\right)$ and the ac and dc components of force-feedback voltage $\left(\mathrm{V}_{\mathrm{ZnO}}\right)$ were recorded, respectively, as a function of distance between the probe and the substrate using the COIFM during both approach and retraction of the sample. ${ }^{47,53,54}$ Using the built-in digital-to-analog converter of the controller in conjunction with a high-voltage amplifier, the sample movement was controlled with the resolution of $\sim 30 \mathrm{pm}$ in the $\mathrm{z}-$ direction, sufficient to control the gap between the two surfaces smaller than water diameter $(\sigma), 0.275$ nm. 
The measurements were performed at the tip-speeds of $3 \mathrm{~nm} / \mathrm{sec}$ under the lateral modulation of the sample at a frequency of $100 \mathrm{~Hz}$ and at an amplitude of $1 \mathrm{~nm}$. The relative humidity was set to $30 \%$ by placing the COIFM system in an acrylic box with one entrance for water vapor and another for dry nitrogen gas. ${ }^{54}$ Room temperature was kept at a consistent $22^{\circ} \mathrm{C}$. The thermo-hygro recorder was placed in close proximity to the head unit to ensure that the humidity and temperature values were most accurate for experimental conditions and to ensure that any localized heating due to the electronics would be minimal. The measurements were performed after approximately one hour from the point of change to allow sufficient saturation time. This saturation time was implemented in order to attain equilibrium between water molecules in the vapor phase and those in other possible phases such as chains. The feedback control parameters, time constant and gain, were manually adjusted for the optimal feedback condition by applying square wave ac signal with a frequency of $10 \mathrm{~Hz}$ as a set-point voltage of the feedback loop before each experiment, as described in our previous report. ${ }^{53}$

\section{Results and Discussion}

Figure 1 shows representative force-distance curves taken at a relative humidity of $30 \%$ and a temperature of $22^{\circ} \mathrm{C}$. The curves exhibit sawtooth-like oscillatory patterns in both normal (open circles) and lateral frictional (solid circles) forces. When we measured the force-distance curves with a new tip at a new location, these periodic oscillatory forces were still

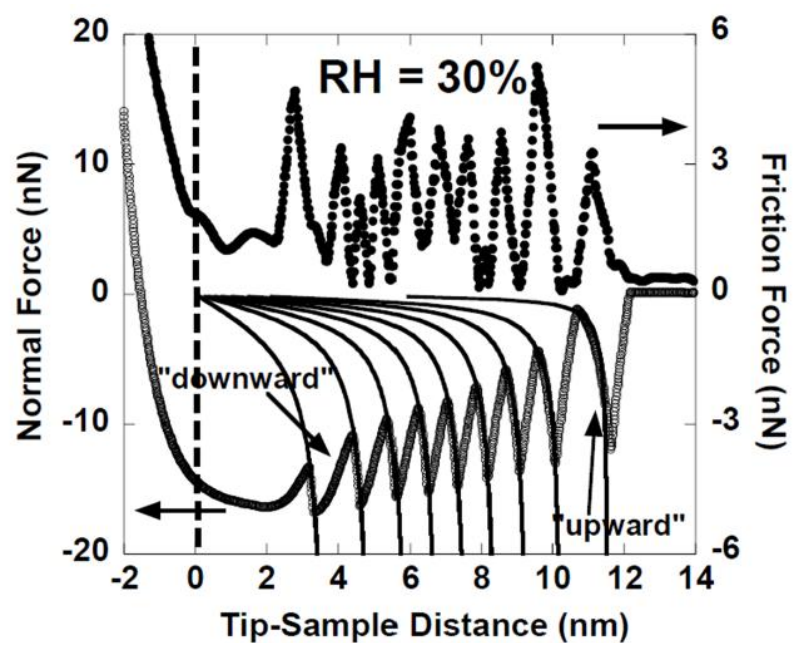

Figure 1. Interfacial force-distance curves for normal force (solid circle) and friction force (open circle) taken at a relative humidity of $30 \%$ at a tip-approaching speed of $3 \mathrm{~nm} / \mathrm{sec}$ while modulating the sample laterally with an amplitude of $\sim 1 \mathrm{~nm}$ at the frequency of $100 \mathrm{~Hz}$. The solid lines represent fitting curves with an altered entropic chain model-the freely-jointed chain (FJC) model-for each layering structure. 
observed, even at the first attempt, without any prior repeated measurement. The zero-point position for the $\mathrm{x}$-axis on the force-distance graph was defined as the point where the friction force increased sharply before the rapid increase of normal force, as marked with a vertical dashed line in Fig. 1. Sudden contact between a solid tip and a solid surface is known to create an abrupt increase in friction force. ${ }^{62}$ The negative distance means the tip position below the surface due to the elastic indentation. It is important to note that the observed oscillatory behavior is not related to the stiffness of the tip or the sample surface as they are too hard to generate such compliances during the tip-excursion. The compliance of the tip material is estimated to be just $\sim 20$ pm using a Hertzian model ${ }^{62}$ with the known stiffness of amorphous silicon dioxide around $70 \mathrm{GPa}^{63}$ for the applied force of $10 \mathrm{nN}$.

The nonlinear oscillatory patterns started near the distance $d=12 \mathrm{~nm}$, and thereafter each period consisted of upward and downward portions as the tip-sample distance became smaller. Since the height of elongated water structures ranged between $2-10 \mathrm{~nm}$ on an oxidized silicon surface, ${ }^{47}$ the onset distance of $12 \mathrm{~nm}$ can be related to the merging distance between the water structures on both tip and sample surfaces. It is not necessary for both surfaces to be applied by any strong electric field ${ }^{12}$ or to be fully filled with water layers less than $\sim 6 \mathrm{~nm}$ each at RH $30 \%$. Because the merging meniscus formation point decreased and the oscillatory forces gradually smeared out with the humidity, ${ }^{47}$ water films appear to form only through coalescence of elongated structures at higher humidities $(>60 \%)$.

The upward force (marked in Fig. 1) increased rapidly and then tapered slightly at the top to form a rising-shaped ( ) curve; while the downward force curve ran in a near-straight line at the formation stage of the water junction, but grew more sigmoidal shaped $(f)$ as the distance became smaller. A downward portion between two subsequent bundles of water chains gradually changed as the distance decreased, suggesting that the observed oscillatory forces are different from the mechanical instabilities of the 
meniscus, such as pinning/unpinning of the contact line (of which the transition would be abrupt), ${ }^{64}$ or a coupling between evaporation/condensation of vapor (which are highly unstable phenomena). ${ }^{65}$ We also excluded the instability of the feedback loop as the origin of the observed oscillatory forces because the feedback signal is stable even with such abrupt changes in the square wave in our routine feedback optimization procedure (see Experimental Section). The oscillatory forces had amplitudes of 3-12 $\mathrm{nN}$ in normal force channel, and periodicities of $1.04 \pm 0.25 \mathrm{~nm}$ and $0.94 \pm 0.16 \mathrm{~nm}$ for valley-valley and for peak-peak distances, respectively. These periodicities are roughly three diameters of water.

Instrumental artifacts (e.g. the ringing of the cantilever, feedback loop, mechanical loop of the system or laser interference, etc.) were also excluded as the possible origin of the oscillatory forces. This is because the periodicity in frictional force is the same with that in normal force. Considering the high selectivity of frequency in the lock-in technique, it is extremely unlikely that such artifacts generated the same periodicity in ac signal (friction force) and de signal (normal force). While they are consistent with the periodicity of $0.6-1.2 \mathrm{~nm}$ (2-4 water diameters) previously reported in the water bridge at the relative humidity of $15 \%,{ }^{49}$ they do not agree with those found in earlier studies at the solid-liquid interface. $^{23,44,50-52,66}$ These oscillatory forces appear to be unique characteristics of water in air because they have not been observed with the menisci formed by soft materials such as hydrocarbon films and biofilms. ${ }^{57-59}$ The unique periodicities $(\sim 1 \mathrm{~nm})$ in this study suggest that when the vapor, meniscus, and solid co-exist, the structure of confined water is different from the structure at the interface between bulk water and a solid surface. The gradual force increase and decrease during the periodicities suggest that the observed force-distance curves were generated not by a single evaporation or condensation process, but by an equilibrium process between evaporation (from meniscus to vapor) and condensation (from vapor to meniscus). 
The nonlinear behavior in the upward portion of each period leads to the use of an FJC model as a means to analyze the water structure in the nanoscopic meniscus. Because the magnitude of the measured force $(\sim 15 \mathrm{nN})$ is larger by a factor of hundreds than a typical single FJC chain force $\left(\frac{k_{B} T}{\sigma} \sim 10 \mathrm{pN}\right)$ in single molecular pulling experiments (e.g. polymethacrylic acid $\left.{ }^{57}\right)$, the water structure must be made of hundreds of chains. As a way to describe this force behavior, the original FJC model was altered from its single-chain expression by including a parameter $n$ related to the number of chains as follows:

$d=l \cdot \sigma \cdot\left[\operatorname{coth}\left(\frac{\left|f_{\text {normal }}\right|}{n} \cdot \frac{\sigma}{k_{B} T}\right)-\left(\frac{n}{\left|f_{\text {normal }}\right|} \cdot \frac{k_{B} T}{\sigma}\right)\right]$

where $d$ is the distance between the two surfaces; $l$ is the number of water joints; $\sigma$ is the chain unit; $k_{B}$ is the Boltzmann constant; and $T$ is temperature. Although the FJC models were developed for a single strand of chain structure, ${ }^{68}$ they have been successfully applied to a system with multiple chains. ${ }^{69}$ The "altered FJC model" is conceptually analogous to a particle attached to a spring when it is released toward its resting position after being stretched. The increase of fluctuation (entropy increase) due to the release of the particle lowers the spring force. Similarly, in the altered FJC model, the orientation fluctuations result in a lower force $(\sim 0 \mathrm{nN})$ along the chain. When the stretching length of the inextensible chain approaches the total contour length, the force becomes higher due to the increase of orientational ordering along the chain.

We applied this altered FJC model to the upward portion $f_{\text {normal }}$ in each period in Fig. 1 with the chain unit of $\sigma=0.275 \mathrm{~nm}$ and two free fitting parameters, the number of water chains $(n)$ and chain length (l). Fig. 1 shows excellent matching between each upward curve (open circles) and the altered FJC 
model (the solid lines). When a linear fitting was performed on each upward curve for comparison, it was shown to be less effective than the altered FJC fitting. As a representative example shown in Fig. 2, the two methods were compared through the correlation value $(R)$ between the measured upward data and the fitting equation (1) in the distance range between $8.9 \mathrm{~nm}$ and $9.1 \mathrm{~nm}$. The $R$ value of the altered FJC fitting is 0.9932 , whereas the $\mathrm{R}$ value of the linear fitting is 0.9818 . This comparison was repeated for all the upward curves. The inset of Fig. 2 shows how the $R$ value changes with distance for both the altered FJC

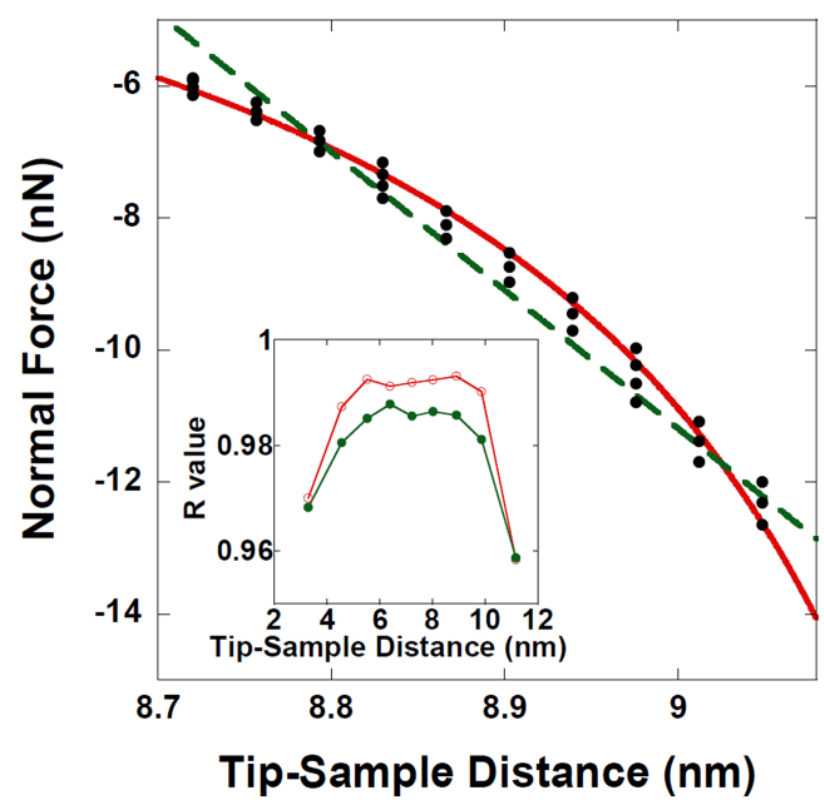

Figure 2. A comparison of fitting methods between linear fitting (green dashed line) and the FJC fitting model (red solid line). (inset) Correlation coefficient (R) vs. tip-sample distance for the FJC fitting (open red circles) and linear fitting (closed green circles). and linear fittings. The altered FJC model fitting has a higher correlation coefficient in the middle distance region, though the coefficients are similar at the lowest and highest tip-sample distances. This result shows that the chain model is a better model than the linear spring model in describing the observed force behavior in each upward portion in Fig 1. This comparison is interesting in that many recent studies viewed the confined water in nanoscopic junctions as a three-dimensional hydrogenbonded network structure or "ice-like structure".,9,44,70,71 The linear fitting or the linear enthalphic spring model is more relevant to the ice-like structure due to the extensive cross linking within the network structure. However, our mechanical response measurement to external forces suggests that the viscous and sticky layers of water could be made of bundles of water chains rather than an ice-like structure in an ambient condition at the low relative humidity of $30 \%$. 
It is important to note that the distances for all chains in a bundle are equal for our FJC analysis. Since the tip end is curved (not truncated) with the tip-radius $(r) \sim 10 \mathrm{~nm}$. These equal distances indicate that the curved-tip area is made of crystallite terraces. ${ }^{49,72}$ Each chain bundle was confined between the top terrace of the tip and the oxidized silicon surface in parallel. Because the solid lines meet at the zero distance all together in Fig. 1, the contact area of the water bundle must stay smaller than the total area of the top terrace during multilayer transitions. This requirement leads to the condition that, for the thickest water bundle with $n \sim 150$, the interchain distance needs to stay smaller stay smaller than 0.35 $\mathrm{nm}\left(\sim \sqrt{\frac{2 \pi r h}{n}}\right)$ on the top-terrace with the width $(w)$ of $\sim 5.0 \mathrm{~nm}(\sim 2 \sqrt{2 r h})$ and the monoatomic step height $(h)$ of $\sim 0.3 \mathrm{~nm} .^{47}$ This estimation suggests that the water chains can exist as a highly packed water bundle with the interchain distance close to the diameter of water $(\sigma \sim 0.3 \mathrm{~nm})$. An increase in tip-radius results in more oscillations due to an increase in the terrace width. The larger number of oscillations observed in our earlier report ${ }^{47}$ can be explained with the use of a tip with a larger tip-radius.

This chain bundle model along with the tip-radius effect can also explain the smearing of the oscillatory patterns in force-distance curves at higher relative humidity values above $\sim 60 \%$, observed in our previous report. ${ }^{47}$ As the water bundle extends beyond the top-terrace of the tip at a higher relative humidity, the distances can no longer be equal. The dependence of these oscillatory forces on the molecular length scale (see Equation (1)) can create the interferences between chains and chains within a thick bundle at a higher relative humidity. This humidity dependent smearing effect strongly supports the idea that the oscillatory forces originated from chain-like water structures. This result also indicates that the pitch-and-roll alignment process (see Experimental section) was successful in vertically aligning the tip to the sample surface. 
Interestingly, the water bundle experiences multiplelayering transitions (without single-layer transitions) from $l=42$ chain units to $l=14$ chain units $(42 \rightarrow 38 \rightarrow 34 \rightarrow 31 \rightarrow 28 \rightarrow 25 \rightarrow 22 \rightarrow 18 \rightarrow 14)$ when $l$ and $n$ are rounded to the closest integers, as the tip approaches the surface (Fig. 1). The result indicates that as the distance between the tip and the substrate decreases, the confined water molecules favor multilayer transitions to stepwise single-layer transitions. Since confined water is in thermodynamic equilibrium with water monomers in the air, its

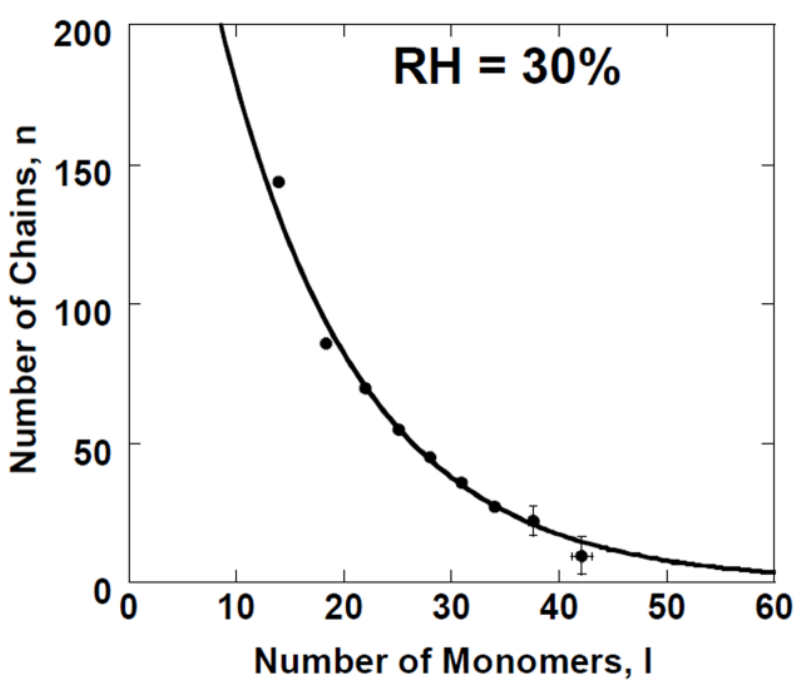

Figure 3. Number of chains (solid circles) with respect to the number of monomers in each layering structure. The solid line is a fitting curve ( $n$ with $l$ ) and its extrapolations. In this data set, $(n, l), n_{0}$ and $\lambda$ were found to be 389.40 and 12.86 , respectively.

structure can result from a two-state system (monomers and their aggregate). According to the onedimensional thermodynamic self-assembly model, ${ }^{1,3}$ the number $(n)$ of the aggregate depends on its size (l) exponentially with a decay length $(\lambda)$. Based on this idea, the extracted numbers of chains $(n)$ were plotted as a function of the chain length $(l)$ as shown in Fig. 3. The solid line represents a curve fitting of the data with an exponential function $n=n_{0} \cdot e^{-l / \lambda}$, showing that $n$ depends on $l$ exponentially with a characteristic decay number $(\lambda)$ of 12.86 . This result indicates that the size distribution of layering structures follows the theoretical size distribution of self-assembled aggregates (micellization). ${ }^{1}$ The decay length is known to be dependent on the total concentration of molecules $(C)$ in mole fraction units and the strength of hydrogen bonding $(\gamma)$ between two water molecules in the unit of the thermal energy $k_{B} T$ by $\lambda=\sqrt{C e^{\gamma}}{ }^{5}$ The $\gamma$ is determined to be 9.97 for $\lambda=12.86$ and $C=7.75 \times 10^{-3}(\mathrm{RH}=30 \%)$. This hydrogen-bond strength $9.97 k_{B} T$ inside the water junction is consistent with the known literature value $9 k_{B} T$ of hydrogen bond strength in bulk water. ${ }^{3}$ This consistency suggests that the observed chain-like 
water structure results from the one-dimensional self-assembly of water monomers and their resultant ordering through hydrogen bonding.

The possibility that the observed oscillatory forces were generated by other contaminant molecules is quantitatively examined, based on the knowledge about $n$ and $l$ in Fig. 3. If the above fitting is assumed to be made by the numbers of contaminant chains $\left(n_{\text {cont }}\right)$ and the chain length $\left(l_{\text {cont }}\right)$, the two fitting parameters, $l_{\text {cont }} \cdot \sigma_{c o n t}$ and $\frac{\sigma_{c o n t}}{n_{c o n t} k_{B} T}$ are equal to $l \cdot \sigma$ and $\frac{\sigma}{n k_{B} T}$, respectively: $l \cdot \sigma=l_{\text {cont }} \cdot \sigma_{c o n t}$ and $\frac{\sigma}{n k_{B} T}=\frac{\sigma_{c o n t}}{n_{c o n t} k_{B} T}$. These relations lead to $n=\frac{n_{c o n t} \sigma}{\sigma_{c o n t}}$ and $l=\frac{l_{\text {cont }} \sigma_{c o n t}}{\sigma}$. When $n$ and $l$ are inserted into the exponential function $n=n_{0} \cdot e^{-l / \lambda}$, the new exponential relation is obtained as $n_{\text {cont }}=n_{\text {cont }, 0} e^{-\frac{l_{c o n t}}{\lambda_{\text {cont }}}}$ with $n_{\text {cont }, 0}=\frac{n_{0} \sigma_{\text {cont }}}{\sigma}$ and $\lambda_{\text {cont }}=\lambda \frac{\sigma}{\sigma_{\text {cont }}}$. According to self-assembled micellization ${ }^{1}, \lambda=\sqrt{C e^{\gamma}}$ and $\lambda_{\text {cont }}=\sqrt{C_{\text {cont }} e^{\gamma_{\text {cont }}}}$ where $\gamma_{\text {cont }} k_{B} T$ represents the interaction between segments. When $\lambda$ and $\lambda_{\text {cont }}$ are inserted into $\lambda_{\text {cont }}=\lambda \frac{\sigma}{\sigma_{\text {cont }}}$, the expected contamination mole fraction $C_{c o n t}$ can be estimated with the following equation:

$$
C_{\text {cont }}=\left(\frac{\sigma}{\sigma_{\text {cont }}}\right)^{2} C e^{\gamma-\gamma_{\text {cont }}}
$$

Most ambient species except water are nonpolar; therefore, their interaction is significantly weaker than that of water. For nonpolar molecules of comparable size such as methane or carbon dioxide, $C_{\text {cont }} \cong C e^{\gamma}$ because $\sigma_{\text {cont }} \sim \sigma$ and $\gamma_{\text {cont }}<<\gamma$. The mole fraction $\mathrm{C}_{\text {cont }}$ is estimated to be $\sim 60$ when $C=7.75 \times 10^{-3}$ and $\gamma=9$. Since the mole fraction cannot be larger than 1 , it is impossible that such 
nonpolar molecules generate the observed larger force oscillations. When chains are hypothetically assumed to be formed by hydrogen bonded contaminants (i.e. $\gamma_{\text {cont }}=\gamma$ ) in the ambient condition, the equation (2) predicts that $C_{\text {cont }}$ would be larger than $0.1 \times C$ because the size of molecule $\sigma_{\text {cont }}$ would be smaller than the observed periodicity (i.e., 3-4 times water diameters). However, no report about ambient gas populations (e.g. ref 73) supports such a highly concentrated hydrogen bonded gas whose size is a few times larger than water diameter. The highest possible organic contaminant is reported to be methane whose ambient concentration is $10^{-4}$ times smaller than water concentration. ${ }^{73}$ Even when we hypothetically assume a high concentration of polar contaminants such as ethanol or methanol, it is unlikely that those alcohols form chain structures through "head-to-tail" hydrogen bonding because of the lack of hydrogen bonding moieties at both ends. Also, the experiment was performed under the wellcontrolled flow of mixed gases of dry nitrogen (purity 99.9\%) and pure DI water mixing (see Experimental section). The performed x-ray photoelectron spectroscopy (see supplemental materials ${ }^{57}$ ) on the cleaned sample surface did not show any perceivable contamination. Therefore, we excluded the possibility that any molecules other than water molecules created the observed oscillatory forces.

Finally, we calculated viscosity $(\eta)$ at the peak friction from the following equation ${ }^{53}$ :

$$
\eta \cong \frac{d}{A x_{\text {mod }} \omega} f_{\text {friction }}
$$

where $x_{\text {mod }}$ is the modulation amplitude, and $\omega$ is the angular frequency. The contact area $A$ was calculated from the sectional area $\left(n \sigma^{2}\right)$, assuming that each chain takes the area of $\sigma^{2}$. In this model, the friction force results from the viscous force due to the lateral translational motion of the tip-surface with respect to the substrate surface. The viscosity $\eta$ at the peak friction of $3 \mathrm{nN}$ was estimated to be $2.7 \times 10^{7}$ $P a \cdot s$ with $d=9.5 \mathrm{~nm}, \sigma=0.275 \mathrm{~nm}, x_{m o d}=1 \mathrm{~nm}, \omega=2 \pi \times 100 \mathrm{~s}^{-1}$ and $n=22$. This value is approximately 
$3.1 \times 10^{10}$ times higher than the known bulk water viscosity value of $8.6 \times 10^{-4} \mathrm{~Pa} \cdot \mathrm{s}$. When the same viscosity $\eta$ is estimated with an alternative model, ${ }^{74}$ it is $2.6 \times 10^{5} \mathrm{~Pa} \cdot \mathrm{s}$, which is still far higher than the bulk water by a factor of $3.0 \times 10^{8}$. This higher viscosity value agrees with the earlier reports about viscous and sticky layers of water in ambient conditions. ${ }^{4,9,10,40,41,42}$ Again, this agreement supports the idea that the observed chain-like structure and its high viscous property originated from water molecules rather than unspecific and unknown contaminants.

Recent inexplicable observations of meniscus heights, far larger than what the Kelvin equation predicts at a lower humidity, can be explained with the stable chain-like water structures. ${ }^{37,38}$ The chain structure is also able to explain the observed slow evaporation rates, ${ }^{37,43}$ elongated structures, ${ }^{46,47}$ and the layer nucleation timescales, ${ }^{45}$ which cannot be predicted with the bulk-water properties. Since this chain-like water structure is connected linearly, the energy needed to break the bonding would be approximately half of the tetrahedral-ice structure. This half-dissociation energy also appeared in the sub-elimination of water molecules from the ice surface studies in earlier studies. ${ }^{45}$ Recent observations of higher viscosity values of water confined between tip and sample surface in air ${ }^{40-42}$ can be associated with the chain-like structure. The observed sensitive dependence of friction force on distance strongly suggests that the force-feedback technique can contribute to the resolution of recent conflicting claims ${ }^{75-78}$ about the water viscosity through separation of the force measurement from the distance control.

Experiments and molecular dynamics simulations have also found that the chain-like structures exist within hyrdophobic carbon nanotubes ${ }^{79-84}$ and narrow hydrophobic pores. ${ }^{85-88}$ Our finding of chain-like structures in air suggests that the chain-like water structure can exist within gaseous hydrophobic environments such as in air as well as within solid hydrophobic cylinders such as nanotubes. In both cases, it appears that the chain-like water structure is one of energetically favorable phases of water although it loses lots of its entropy from bulk water phase due to the confinement. Considering the 
capability of COIFM method in measuring forces with high resolution and controlling gap-distance with high precision, the water meniscus in air may present unprecedented model systems to study biological functions such as water channels through transmembrane pores. For these studies, the use of a calibrated spring constant instead of using a nominal spring constant would provide more precise force values as the nominal constant may lead to force error by $20-30 \%$ on a given wafer ${ }^{89}$. As seen in the transition periodicity increases under the lateral modulation (thus the increases of the effective temperature), ${ }^{47}$ oscillatory force measurements at various controlled temperatures would allow better understanding of chain-like water structures and their kinetically activated processes.

\section{Conclusion}

We have observed large sawtooth-like oscillatory forces generated by confined water under lateral modulation between two hydrophilic surfaces using the newly developed COIFM. The COIFM data suggest that water molecules self-assembled to form chain-like structures in a nanoscopic space between two hydrophilic surfaces in air. In addition, the self-assembly mechanism eliminated the possibility that molecules other than water molecules in ambient air created such large oscillatory forces. The viscosity of the chain-like water was estimated to be $10^{8}-10^{10}$ times greater than that of bulk water. Many recent inexplicable observations, including positive stable Kelvin radii, slow evaporation rates, and long nucleation timescales can be explained by the stable chain-like water structuring found in this study. The knowledge obtained through this study has the potential to greatly benefit a variety of fields, including many biological studies.

Acknowledgement: This research was supported by NSF DMR-1126854, NSF DBI-0852886, NSF EPSCOR Startup Augmentation Funding, the Research Corporation Single-Investigator (Cottrell 
This document was originally published by American Institute of Physics (AIP) in The Journal of Chemical Physics. Copyright restrictions may apply. DOI: $10.1063 / 1.4816818$

College Science Award No. CC7041/7162), and the Korean Ministry of Education, Science and Technology. 


\section{REFERENCES}

1. C. Tanford, The Hydrophobic Effect: Formation of Micelles and Biological Membranes, second edition (Wiley, New York, 1980).

2. S. K. Pal and A. H. Zewail, Chem. Rev. 104, 2099-2123 (2004).

3. P. Nelson, Biological Physics: Energy, Information, Life, updated edition (W.H. Freeman, New York, 2008).

4. A. Verdaguer, G. M. Sacha, H. Bluhm, and M. Salmeron, Chem. Rev. 106, 1478-1510 (2006).

5. J. N. Israelachvili, Intermolecular and Surface Forces, second edition (Academic Press INC, San Diego, CA, 1991).

6. V. N. Paunov, R. I. Dimova, P. A. Kralchevsky, G. Broze, and A. Mehreteab, Colloid Interface Sci. 182, 239-248 (1996).

7. K. R. Trethewey and K. R. Chamberlain, Corrosion for Science and Engineering, second edition (Longman Group, England, 1995).

8. N. E. Dorsey, Properties of Ordinary Water-Substance in All its Phases: Water-Vapor, Water, and All the Ices (Hafner Pub. Co., New York, 1940), Vol. 81.

9. J. Hu, X.-D. Xiao, D. F. Ogletree, and M. Salmeron, Science 268, 267-269 (1995).

10. J. Hu, X.-D. Xiao, and M. Salmeron, Appl. Phys. Lett. 67, 476-478 (1995).

11. R. Zangi and A. E. Mark, Phys. Rev. Lett. 91, 025502 (2003). 
12. G. M. Sacha, A. Verdaguer, and M. Salmeron, J. Phys. Chem. B 110, 14870 (2006).

13. B. Vonnegut, J. Appl. Phys. 18, 593-595 (1947).

14. K. P.Stevenson, G. A. Kimmel, Z. Dohnalek, R. S. Smith, and B. D. Kay, Science 283, 1505-1507 (1999).

15. H. Tanaka and K. Koga, Bull. Chem. Soc. Jpn. 79, 1621-1644 (2006).

16. M. F. Chaplin, Biophys. Chem. 83, 211-221 (1999).

17. J. J. Gilijamse, A. J. Lock, and H. J. Bakker, Proc. Natl. Acad. Sci. 102, 3202-3207 (2005).

18. K. Koga, G. T. Gao, H. Tanaka, and X. C. Zeng, Nature 408, 564-567 (2000).

19. E. A. Jagla, Phys. Rev. Lett. 88, 245504 (2002).

20. R. Zangi and A. E. Mark, J. Chem. Phys. 120, 7123-7130 (2004).

21. R. Zangi and A. E. Mark, J. Chem. Phys. 119, 1694 (2003).

22. R. Zangi, J. Phys. Condens. Matter 16, S5371-S5388 (2004).

23. M. J. Higgins, M. Polcik, T. Fukuma, J. E. Sader, Y. Nakayama, and S. P. Jarvis, Biophys. J. 91, 2532-2542 (2006).

24. S. Leikin, V. A. Parsegian, D. C. Rau, and R. P. Rand, Annu. Rev. Phys. Chem. 44, 369-395 (1993).

25. P. M. Wiggins, MicroBio. Rev. 54, 432-449 (1990).

26. A. B. Naim, Biophys. Chem. 105, 183-193 (2003). 
27. K. Maurata, Y. Fujii, N. Enomoto, M. Hata, T. Hoshino, and M. Tsuda, Biophys. J. 79, $982-991$ (2000).

28. J. Katsaras and K. R. Jeffrey, Europhys. Lett. 38, 43-48 (1997).

29. H. Sakuma, K. Otsuki, and K. Kurihara, Phys. Rev. Lett. 96, 046104 (2006).

30. U. Raviv, S. Giasson, J. Frey, and J. Klein, J. Phys. Condens. Matter 14, 9275-9283 (2002).

31. K. Koga, G. T. Gao, H. Tanaka, and X. C. Zeng, Nature 412, 802-805 (2001).

32. A. Pertsin and M. Grunze, J. Phys. Chem. B 108, 1357-1364 (2004).

33. K. Koga and H. Tanaka, J. Chem. Phys. 122, 104711 (2005).

34. N. Giovambattista, P. J. Rossky, and P. G. Debenedetti, Phys. Rev. E 73, 041604 (2006).

35. B. V. Derjaguin and L. Landau, Acta. Physico. Chemica. URSS. 14, 633 (1941).

36. E. J. W. Verwey and J. T. G. Overbeck, Theory of the Stability of Lyophobic Colloids (Elsevier, New York, 1948).

37. B. L. Weeks, M. W. Vaughn, and J. J. DeYoreo, Langmuir 21, 8096-8098 (2005).

38. M. Schenk, M. Futing, and R. Reichelt, J. Appl. Phys. 84, 4880-4884 (1998).

39. V. V. Yaminsky, S. Ohnishi, and B. W. Ninham, Handbook of Surfaces and Interfaces of Materials, Eds.H. S. Nalwa (Academic Press, New York, 2001), Vol. 4, p. 132-226.

40. K. B Jinesh and J. W. M. Frenken, Phys. Rev. Lett. 96, 166103 (2006). 
41. J. W. M. Goertz, J. E. Houston, and X. -Y. Zhu, Langmuir 23, 5491-5497 (2007).

42. R. C. Major, J. E. Houston, M. J. McGrath, J. I. Siepmann, and X. - Y. Zhu, Phys. Rev. Lett. 96, 177803 (2006).

43. J. Grobelny, N. Pradeep, D. -I. Kim, and Z. C. Yang, Appl. Phys. Lett. 88, 091906 (2006).

44. T. -D. Li, J. Gao, R. Szoszkiewicz, U. Landman, and E. Riedo, Phys. Rev. B 75, 115415 (2007).

45. J. G. Davy and G.A Somorjai, J. Chem. Phys. 55, 3624-3636 (1971).

46. K. Chiba, R. Ohmori, H. Tanigawa, T. Yoneoka, and S. Tanaka, Fusion Eng. Des. 49, 791-797 (2000).

47. B. I. Kim, J. A. Rasmussen, and E. J. Kim, Appl. Phys. Lett. 99, 201902 (2011).

48. Z. Liu, Z. Li, H. Zhou, G. Wei, Y. Song, L. Wang, Micron 36, 525-531 (2005)

49. H. Choe, M. -H. Hong, Y. Seo, K. Lee, G. Kim, Y. Cho, J. Ihm, and W. Jhe, Phys. Rev. Lett. 95 , $187801(2005)$.

50. S. P Jarvis, T. Uchihashi, T. Ishida, H. Tokumoto, and Y. Nakayama, J. Phys. Chem. B 104, 60916094 (2000).

51. S. Jeffery, P. M. Hoffmann, J. B. Pethica, C. Ramanujan, H. Ö. Özer, and A. Oral, Phys. Rev. B 70, 054114 (2004).

52. T. Uchihashi, M. Higgins, Y. Nkayama, J. E. Sader, and S. P. Jarvis, Nanotechnology 16, S49-S53 (2005). 
53. J. R. Bonander and B. I. Kim, Appl. Phys. Lett. 92, 103124 (2008).

54. B. I. Kim, J. R. Bonander, and J. A. Rasmussen, Rev. Sci. Instrum. 82, 053711 (2011).

55. B. I. Kim, Cantilever-Based Optical Interfacial Force Microscopy, in: A. Meghea (Eds.), Molecular Interactions, Intech, ISBN: 978-953-51-0079-9, (InTech, New York, 2012), p.125 -144.

56. B. I. Kim, L. Smith, T. Tran, S. Rossland, E. Parkinson, AIP Advances 3, 032126 (2013).

57. B. I. Kim and R. D. Boehm, Micron 43, 1372-1379 (2012).

58. B. I. Kim and R. D. Boehm, Ultramicroscopy 125, 29-34 (2013).

59. B. I. Kim and R. D. Boehm, Scanning 35, 59-67 (2013).

60. Bruker, Probes and Accessories (Bruker, Camarillo, CA, 2012), p. 126-127.

61. See supplementary material at [URL will be inserted by AIP] for x-ray photoelectron spectroscopy data used to check for possible foreign impurities on the cleaned, oxidized silicon wafer surface.

62. B. I. Kim, S. Lee, R. L. Guenard, L. C. Fernandez-Torres, S. S. Perry, P. Frantz, and S. V. Didziulis, Surf. Sci. 481, 185-197 (2001).

63. A. Khan, J. Philip, and P. Hess, J. Appl. Phys. 95, 1667-1672 (2004).

64. J. Crassous, E. Charlaix, and J. L. Loubet, Phys. Rev. Lett. 78, 2425-2428 (1997).

65. N. Maeda, J. N. Israelachvili, and M. M. Kohonen, Proc. Natl. Acad. Sci. USA, 100, 803-808 (2003). 
66. S. P. Jarvis, T. Ishida, T. Uchihashi, Y. Nakayama, and H. Tokumoto, Appl. Phys. A: Mater. Sci. Process. 72, S129-S132 (2001).

67. C. Ortiz and G. Hadziioannou, Macromolecules 32, 780-787 (1999).

68. R. Phillips, J. Kondev, and J. Theriot, Physical Biology of the Cell, first edition (Garland Science, New York City, NY, 2008), p. 313-317.

69. T. Sulchek, R. W. Friddle, and A. Noy, Biophys. J. 90, 4686-4691 (2006).

70. D. B. Asay and S. H. Kim, J. Chem. Phys. 124, 174712 (2006).

71. D. B. Asay and S. H. Kim, J. Phys. Chem. B 109, 16760-16763 (2005).

72. T. Arai and M. Tomitori, Appl. Phys. Lett. 86, 073110 (2005).

73. M. Pidwirny, Atmospheric composition. Eds. D. Budikova and K. Vranes, In: Encyclopedia of Earth. Eds. Cutler J. Cleveland (Washington, D.C.: Environmental Information Coalition, National Council for Science and the Environment). (First published in the Encyclopedia of Earth July 14, 2010; Last revised Date July 14, 2010.)

74. P. J. Feibelman, Langmuir 22, 2136.-2140 (2006).

75. U. Raviv, S. Perkin, P. Laurat, and J. Klein, Langmuir 20, 5322-5332 (2004).

76. U. Raviv, P. Laurat, and J. Klein, Nature 413, 51-54 (2001).

77. U. Raviv and J. Klein, Science 297, 1540-1543 (2002).

78. S. H. Khan, G. Matei, S. Patil, and P. M. Hoffmann, Phys. Rev. Lett. 105, 106101 (2010). 
79. P. Ball, Chem. Rev.108, 74-108 (2008).

80. D. J. Mann and M. D. Halls, Phys. Rev. Lett. 90, 195503-195506 (2003).

81. C. Dellago, M. M. Naor, and G. Hummer, Phys. Rev. Lett. 90, 105902-105904 (2003).

82. G. Hummer, J. C. Rasaiah, and J. P. Noworyta, Nature 414, 188-190 (2001).

83. R. J. Mashl, S. Joseph, N. R. Aluru, and E. Jakobsson, Nano Lett. 3, 589-592 (2004).

84. O. Byl, J.-C. Liu, Y. Wang, W.-L. Yim, J. K. Johnson, and J. T. Yates Jr., J. Am. Chem. Soc. 128, 12090-12097 (2006).

85. H. Luecke, B. Schobert, H.-T. Richter, J.-P. Cartailler, and J. K. Lanyi, J. Mol. Biol. 291, 899-911 (1999).

86. H. J. Sass, G. Bu“ldt, R. Gessenich, D. Hehn, D. Neff, R. Schlesinger, J. Berendzen, and P. Ormos, Nature 406, 649-653 (2000).

87. J. Baudry, E. Tajkhorshid, F. Molnar, J. Phillips, and K. Schulten, J. Phys. Chem. B 105, 905-918 (2001).

88. S. Grudinin, G. Bu“ldt, V. Gordeliy, and A. Baumgaertner, Biophys. J. 88, 3252-3261 (2005).

89. C. T. Gibson, G. S. Watson, and S. Myhra, Nanotechnology 7, 259-262 (1996). 